\title{
Diffraction at the LHC - antishadow scattering?
}

\author{
S. M. Troshin, N. E.Tyurin \\ Institute for High Energy Physics, \\ Protvino, Moscow Region, 142284 Russia
}

\begin{abstract}
Numerical predictions for the global characteristics of proton-proton interactions are given for the LHC energy. Possibilities for the discovery of the antishadow scattering mode and its physical implications are discussed. PACS numbers: 12.40.Pp; 13.85.Dz; 13.85.Lg
\end{abstract}

\section{Introduction}

Soft hadronic interactions observe a time oscillating pattern in the interest from a high-energy physics community. The peaks of the interest coincide as usual with the beginning of the new machine operation. Nowadays RHIC is preparing for operation and LHC would start to provide first results in the not too distant future. Under these circumstances the interest to experimental and theoretical studies in this field is increasing.

There are many open problems in hadron physics at large distances and their importance has not been overshadowed by the exciting expectations of the new particles discoveries in the newly opening energy range of the LHC.

The most global characteristic of the hadronic collision is the total crosssection and the most important problem here is the nature of the total-cross section rising energy dependence. There are various approaches which provide total cross-section rising with energy but the underlying microscopic mechanism leading to this increase remains obscure. However, the growing understanding how QCD works at large distances could finally lead to a final explanation of this longstanding problem [1]. 
In this connection the TOTEM experiment [2] approved recently at the LHC could be more valuable than just a tool for checking numerous model predictions and background and luminosity estimates. It could have a definite discovery potential and our main goal in this note is to discuss one of the such aspects related to the possible observation of the antishadow scattering mode at the LHC.

\section{When will asymptotics be seen?}

The answer on the above question currently is model dependent. There are many model parameterizations for the total cross-sections using $\ln ^{2} s$ dependence for $\sigma_{t o t}(s)$. This implies the saturation of the Froissart-Martin bound and what is unnatural the presence of the asymptotical contributions already at the very moderate energies. On the other side the power-like parameterizations of $\sigma_{t o t}(s)$ neglect the Froissart-Martin bound considering it as a matter of the distant unknown asymptopia.

It seems that the both approaches are limited and their limitations reflect the real energy range available for the analysis of the experimental data. For example, it is not clear whether the power-like parameterizations respect unitarity limit for the partial-wave amplitudes $\left|f_{l}(s)\right| \leq 1$. We are keeping in mind here only the accelerator data (cosmic ray data will be briefly commented below).

Unitarity is an important principle and the unitarization procedure of some input power-like "amplitude" leads to the complicated energy dependence of $\sigma_{\text {tot }}(s)$ which can be approximated by the various functions depending on the particular energy range under consideration. Moreover, unitarity implies the appearance of the new scattering mode - antishadow (see [3] and references therein). Here we provide numerical estimates at LHC energies based on the $U$-matrix unitarization method [4] and the particular model for $U$-matrix [5] and argue that antishadow mode could be revealed already at the LHC energy $\sqrt{s}=14 \mathrm{TeV}$.

\section{Antishadow scattering at LHC}

In the impact parameter representation the unitarity equation written for the elastic scattering amplitude $f(s, b)$ at high energies has the form

$$
\operatorname{Imf}(s, b)=|f(s, b)|^{2}+\eta(s, b)
$$

where the inelastic overlap function $\eta(s, b)$ is the sum of all inelastic channel contributions. It can be expressed as a sum of $n$-particle production cross-sections at 
the given impact parameter

$$
\eta(s, b)=\sum_{n} \sigma_{n}(s, b) .
$$

Unitarity equation has the two solutions for the case of pure imaginary amplitude:

$$
f(s, b)=\frac{i}{2}[1 \pm \sqrt{1-4 \eta(s, b)}] .
$$

Eikonal unitarization with pure imaginary eikonal corresponds to the choice of the particular solution with sign minus.

In the $U$-matrix approach the form of the elastic scattering amplitude in the impact parameter representation is the following:

$$
f(s, b)=\frac{U(s, b)}{1-i U(s, b)} .
$$

$U(s, b)$ is the generalized reaction matrix, which is considered as an input dynamical quantity similar to eikonal function.

Inelastic overlap function is connected with $U(s, b)$ by the relation

$$
\eta(s, b)=\frac{\operatorname{Im} U(s, b)}{|1-i U(s, b)|^{2}} .
$$

Construction of particular models in the framework of the $U$-matrix approach proceeds the standard steps, i.e. the basic dynamics as well as the notions on hadron structure are used to obtain a particular form for the $U$-matrix.

However, the two unitarization schemes ( $U$-matrix and eikonal) lead to different predictions for the inelastic cross-sections and for the ratio of elastic to total cross-section. This ratio in the $U$-matrix unitarization scheme reaches its maximal possible value at $s \rightarrow \infty$, i.e.

$$
\frac{\sigma_{e l}(s)}{\sigma_{t o t}(s)} \rightarrow 1
$$

which reflects in fact that the bound for the partial-wave amplitude in the $U$ matrix approach is $|f(s, b)| \leq 1$ while the bound for the case of imaginary eikonal is (black disk limit): $|f(s, b)| \leq 1 / 2$.

When the amplitude exceeds the black disk limit (in central collisions at high energies) then the scattering at such impact parameters turns out to be of an antishadow nature. In this antishadow scattering mode the elastic amplitude increases with decrease of the inelastic channels contribution. 
The shadow scattering mode is considered usually as the only possible one. But the two solutions of the unitarity equation have an equal meaning and the antishadow scattering mode could also appear in the central collisions first as the energy becomes higher. The both scattering modes are realized in a natural way under the $U$-matrix unitarization despite the two modes are described by the two different solutions of unitarity.

Appearance of the antishadow scattering mode is consistent with the basic idea that the particle production is the driving force for elastic scattering. Indeed, the imaginary part of the generalized reaction matrix is the sum of inelastic channel contributions:

$$
\operatorname{Im} U(s, b)=\sum_{n} \bar{U}_{n}(s, b)
$$

where $n$ runs over all inelastic states and

$$
\bar{U}_{n}(s, b)=\int d \Gamma_{n} \mid U_{n}\left(s, b,\left.\left\{\xi_{n}\right\}\right|^{2}\right.
$$

and $d \Gamma_{n}$ is the $n$-particle element of the phase space volume. The functions $U_{n}\left(s, b,\left\{\xi_{n}\right\}\right)$ are determined by the dynamics of $2 \rightarrow n$ processes. Thus, the quantity $\operatorname{ImU}(s, b)$ itself is a shadow of the inelastic processes. However, unitarity leads to self-damping of the inelastic channels [6] and increase of the function $\operatorname{ImU}(s, b)$ results in decrease of the inelastic overlap function $\eta(s, b)$ in accord with Eq. (5) when $\operatorname{ImU}(s, b)$ exceeds unity.

Let us consider the transition to the antishadow scattering mode [7]. With conventional parameterizations of the $U$-matrix the inelastic overlap function increases with energies at modest values of $s$. It reaches its maximum value $\eta(s, b=0)=1 / 4$ at some energy $s=s_{0}$ and beyond this energy the antishadow scattering mode appears at small values of $b$. The region of energies and impact parameters corresponding to the antishadow scattering mode is determined by the conditions $\operatorname{Imf}(s, b)>1 / 2$ and $\eta(s, b)<1 / 4$. The quantitative analysis of the experimental data [8] gives the threshold value: $\sqrt{s_{0}} \simeq 2 \mathrm{TeV}$.

Thus, the function $\eta(s, b)$ becomes peripheral when energy is increasing. At such energies the inelastic overlap function reaches its maximum value at $b=$ $R(s)$ where $R(s)$ is the interaction radius. So, beyond the transition threshold there are two regions in impact parameter space: the central region of antishadow scattering at $b<R(s)$ and the peripheral region of shadow scattering at $b>R(s)$.

The region of the LHC energies is the one where antishadow scattering mode is to be presented. It will be demonstrated in the next section that this mode can be revealed directly measuring $\sigma_{e l}(s)$ and $\sigma_{t o t}(s)$ and not only through the analysis of impact parameter distributions. 


\section{Estimates and transition to asymptotics}

We use chiral quark model for the $U$-matrix [5]. The function $U(s, b)$ is chosen in the model as a product of the averaged quark amplitudes

$$
U(s, b)=\prod_{Q=1}^{N}\left\langle f_{Q}(s, b)\right\rangle
$$

in accordance with assumed quasi-independent nature of the valence quark scattering in some effective field. The essential point here is the rise with energy of the number of the scatterers like $\sqrt{s}$ (cf. [5]). The $b$-dependence of the function $\left\langle f_{Q}\right\rangle$ is related to the quark formfactor $F_{Q}(q)$ and has a simple form $\left\langle f_{Q}(b)\right\rangle \propto \exp \left(-m_{Q} b / \xi\right)$, i.e. the valence quarks in the model have a complicated structure with quark matter distribution approximated by the function $\left\langle f_{Q}(b)\right\rangle$.

The generalized reaction matrix (in a pure imaginary case) gets the following form

$$
U(s, b)=i g\left[1+\alpha \frac{\sqrt{s}}{m_{Q}}\right]^{N} \exp (-M b / \xi),
$$

where $M=\sum_{Q=1}^{N} m_{Q}$. Here $m_{Q}$ is the mass of constituent quark, which is taken to be $0.35 \mathrm{GeV}, N$ is the total number of valence quarks in the colliding hadrons, i.e. $N=6$ for $p p$-scattering. The values for the other parameters were obtained in [8]: $g=0.24, \xi=2.5, \alpha=0.56 \cdot 10^{-4}$. These parameters were adjusted to the experimental data on the total cross-sections in the range up to the Tevatron energy. With such small number of free parameters the model is in a rather good agreement with the data [8].

For the LHC energy $\sqrt{s}=14 \mathrm{TeV}$ the model gives

$$
\sigma_{\text {tot }} \simeq 230 \mathrm{mb}
$$

and

$$
\sigma_{e l} / \sigma_{t o t} \simeq 0.67
$$

Thus, the antishadow scattering mode could be discovered at the LHC by measuring $\sigma_{e l} / \sigma_{\text {tot }}$ ratio which is greater than the black disc value $1 / 2$.

However, the LHC energy is not in the asymptotic region yet; the total, elastic and inelastic cross-sections behave like

$$
\sigma_{t o t, e l} \propto \ln ^{2}\left[g\left(1+\alpha \frac{\sqrt{s}}{m_{Q}}\right)^{N}\right],
$$




$$
\sigma_{\text {inel }} \propto \ln \left[g\left(1+\alpha \frac{\sqrt{s}}{m_{Q}}\right)^{N}\right] .
$$

True asymptotical regime

$$
\sigma_{t o t, e l} \propto \ln ^{2} s, \quad \sigma_{i n e l} \propto \ln s
$$

is expected at $\sqrt{s}>100 \mathrm{TeV}$.

Another predictions of the chiral quark model is decreasing energy dependence of the the cross-section of the inelastic diffraction at $s>s_{0}$. Decrease of diffractive production cross-section at high energies $\left(s>s_{0}\right)$ is due to the fact that $\eta(s, b)$ becomes peripheral at $s>s_{0}$ and the whole picture corresponds to the antishadow scattering at $b<R(s)$ and to the shadow scattering at $b>R(s)$ where $R(s)$ is the interaction radius:

$$
\frac{d \sigma_{d i f f}}{d M_{X}^{2}} \simeq \frac{8 \pi g^{*} \xi^{2}}{M_{X}^{2}} \eta(s, 0) .
$$

The parameter $g^{*}<1$ is the probability of the excitation of a constituent quark during interaction. Diffractive production cross-section has familiar $1 / M_{X}^{2}$ dependence which is related in this model to the geometrical size of excited constituent quark.

At the LHC energy $\sqrt{s}=14 \mathrm{TeV}$ the value of the single diffractive inelastic cross-sections is limited by the value

$$
\sigma_{\operatorname{diff}}(s) \leq 2.4 \mathrm{mb}
$$

The above predicted values for the global characteristics of $p p$ - interactions at the LHC differ from the most common predictions of the other models. First of all total cross-section is predicted to be twice as much of the standard predictions in the range 95-120 mb [10] and it also overshoots the existing cosmic ray data. However, extracting proton-proton cross sections from cosmic ray experiments is model dependent and far from straightforward (see, e.g. [9] and references therein). Those experiments measure the attenuation lengths of showers initiated by the cosmic particles in the atmosphere and are sensitive to the model dependent parameter called inelasticity. So the disagreement of the particular model with the cosmic ray measurements means that the data should be recalculated in the framework of this model and in addition assumptions on the energy dependence of inelasticity should be involved also. 


\section{Discussions and conclusion}

The main goal of this note is to point out that the antishadow scattering mode at the LHC can be detected measuring elastic to total cross section ratio which is predicted to be greater than the black disc limit $1 / 2$. The considered model estimates also the total cross section values significantly higher than the values conventional parameterizations provide.

The studies of soft interactions at the LHC energies can lead to the discoveries of fundamental importance. The genesis of hadron scattering with rising energy can be described as transition from the grey to black disc and eventually to black ring with the antishadow scattering mode in the center. It is worth noting that the appearance of the antishadow scattering mode at the LHC implies a somewhat unusual scattering picture. At high energies the proton should be represented as a very loosely bounded composite system and it appears that this system has a high probability to reinstate itself only in the central collisions where all of its parts participate in the coherent interactions. Therefore the central collisions are mostly responsible for elastic processes while the peripheral ones where only few parts of weekly bounded protons are involved result mainly in the production of secondary particles. This leads to the peripheral impact parameter profile of the inelastic overlap function. The above picture would imply interesting consequences for the multiplicities in hadronic collisions, i.e. up to the threshold energy $s_{0}$ the picture will correspond to the fragmentation concept [11] which supposes larger multiplicity for the higher value of momentum transfer. The increase of the mean multiplicity in hadron interactions with $t$ [12] is in agreement with the hadronic experimental data. However, when the energy becomes greater than $s_{0}$ and antishadow mode develops, momentum transfer dependence of multiplicity would change. Loosely speaking the picture described above correspond to the scattering of extended objects at lower energies and transition to the scattering of weakly bounded systems at higher energies. This picture has an illustrative value and is in general compliance with asymptotic freedom of QCD and parton picture.

Finally, we would like to note that the numerical predictions depend on the particular choice of the model for the $U$-matrix, but appearance of the antishadow scattering mode is an inherent feature of the considered approach. 


\section{Acknowledgements}

Authors are grateful to W. Kienzle, A. Krisch, W. Lorenzon and V. Roinishvili for the interesting discussions. This work was supported in part by the RFBR Grant No. 99-02-17995.

\section{References}

[1] A. Martin, CERN-TH.7284/94 Preprint, 1994.

[2] G. Matthiae, In "Future Physics and Accelerators", Edited by M. Chaichian, K. Huitu, R. Orava, World Scientific, 1995, 245.

[3] S. M. Troshin and N. E. Tyurin, Phys. Part. Nucl. 30 (1999) 550.

[4] A. A. Logunov, V. I. Savrin, N. E. Tyurin and O. A. Khrustalev, Teor. Mat. Fiz. 6 (1971) 157 ;

[5] S.M. Troshin and N.E. Tyurin, Nuovo Cim. 106A (1993) 327; Proc. of the Vth Blois Workshop on Elastic and Diffractive Scattering, Providence, Rhode Island, June 1993, p. 387; Phys. Rev. D49 (1994) 4427; Z. Phys. C64 (1994) 311.

[6] M. Baker and R. Blankenbecler, Phys. Rev. 128 (1962) 415.

[7] S. M. Troshin and N. E. Tyurin, Phys. Lett. B 316 (1993) 175.

[8] P. M. Nadolsky, S. M. Troshin and N. E. Tyurin, Z. Phys. C 69 (1995) 131.

[9] M. M. Block, F. Halzen and T. Stanev, hep-ph/9908222 Preprint, 1999.

[10] J. Velasco , J. Perez-Peraza, A. Gallegos-Cruz, M. Alvarez-Madrigal, A. Faus-Golfe, A. Sanchez-Hertz, hep-ph/9910484 Preprint, 1999.

[11] J. Benecke, T. T. Chou, C. N. Yang and E. Yen, Phys. Rev. 188 (1969) 2159; T. T. Chou and C. N. Yang, Phys. Rev. D 50 (1994) 590.

[12] S. M. Troshin, Sov. J. Nucl. Phys. 25 (1977) 472. 